\title{
Review of Extraosseous Applications of Thermal Ablation in the Treatment of Moderate to Severe Large Joint Osteoarthritis
}

\author{
Andrew Tran, BS ${ }^{1}$ David A. Reiter, $\mathrm{PhD}^{2}$ J. David Prologo, $\mathrm{MD}^{3}$ Mircea Cristescu, MD ${ }^{3}$ \\ Felix M. Gonzalez, MD ${ }^{3}$
}

${ }^{1}$ Department of Radiology and Imaging Sciences, Emory University
School of Medicine, Atlanta, Georgia
2 Department of Radiology and Imaging Sciences, and Orthopedics,
Emory University School of Medicine, Atlanta, Georgia
${ }^{3}$ Department of Radiology and Imaging Sciences, Emory University
School of Medicine, Atlanta, Georgia

Address for correspondence Felix M. Gonzalez, MD, Department of Radiology and Imaging Sciences, Emory University School of

Medicine, Atlanta, GA (e-mail: felix.m.gonzalez@emory.edu).

Semin Musculoskelet Radiol 2021;25:745-755.

\begin{abstract}
Osteoarthritis $(\mathrm{OA})$ is the most common joint disease worldwide, leading to significant pain, restricted range of motion, and disability. A gap exists between short- and longterm symptom-relieving therapies. Although arthroplasty is an effective treatment for symptomatic end-stage disease, most patients ultimately do not receive a joint replacement due to suboptimal surgical qualifications, comorbidities, or an aversion

Keywords

- cooled radiofrequency ablation

- arthritis

- hip

- knee

- shoulder
\end{abstract}

Osteoarthritis (OA) is the most common disease process of the large joints, involving $\sim 650$ million adults $>40$ years of age worldwide as of $2020 .^{1}$ The pathophysiology involves degeneration of articular cartilage, the connective tissue responsible for transmitting load across joints. This is accompanied by irreversible bony remodeling: osteophyte overgrowth, subchondral plate thickening, and severe chondral wear. The annual financial impact for caring for this disease, including earnings lost for adults, was reported to have reached $>\$ 300$ billion in 2013 . $^{2}$ Because $\mathrm{OA}$ is a disease associated with aging and metabolic disease, increases in OA-related costs are anticipated as people live longer and as obesity rates continue to climb. ${ }^{3,4}$

Patients with $\mathrm{OA}$ usually present with chronic pain, limited range of motion, and muscle weakness. The typical radiologic signs are joint space narrowing, cyst formation, osteophytes, and subchondral sclerosis. OA typically affects the knee and hip, but it can also significantly affect the shoulder, as seen in $32.8 \%$ of cadaveric and radiologic studies in patients $>60$ years of age. ${ }^{5}$ The clinical management for $\mathrm{OA}$ ranges from activity modification, to intra-articular injection, to surgery. Intra-articular anesthetic corticosteroid injections are aimed to reduce joint inflammation and pain, with a large variation in efficacy ranging from 1 to 24 weeks. ${ }^{6}$ Long treatment durations of corticosteroid injections were also associated with chondrotoxicity, worsening disease progression. ${ }^{7,8}$ Patients eventually turn to surgery for long-lasting pain relief; however, arthroplasty may have complications such as infection, failed implants, and
Issue Theme Musculoskeletal Intervention; Guest Editors, Connie Y. Chang, MD and Mary K. Jesse, MD (c) 2021. Thieme. All rights reserved. Thieme Medical Publishers, Inc., 333 Seventh Avenue, 18th Floor, New York, NY 10001, USA
DOI https://doi.org/ $10.1055 / \mathrm{s}-0041-1735474$. ISSN 1089-7860. 
continued joint pain. The longevity of hip and knee implants was estimated as $58 \%$ and $82 \%$ at 25 -year follow-up, respectively. ${ }^{9}$ The primary symptom of $\mathrm{OA}$ is joint pain that can exist beyond curative surgery. ${ }^{10}$ Joint pain persists beyond 3 to 5 months in $20 \%$ of patients after knee replacement surgery and $10 \%$ of patients after hip replacement surgery. ${ }^{11}$ Thermal neurolysis has evolved as an alternative method of treating the arthritic symptoms among patients who are not surgical candidates for joint replacement or who are experiencing postoperative pain without hardware complications or infection.

Neurolysis, which disrupts pain signal transmissions, is a way to mitigate pain from advanced OA or postsurgical pain. Thermal radiofrequency ablation (TRFA) is a relatively novel treatment method that uses radiofrequency probes to ablate these nerves at temperatures at temperatures $\sim 80$ to $90^{\circ} \mathrm{C}^{12}$ CRFA (cooled radiofrequency ablation) is a type of TRFA but considered more effective because it delivers a greater ablative zone, accounting for the anatomical variability of nerve branches. CRFA has already been used to treat knee pain and shown improved joint function and decreased dependence on pain medication for up to 2 years in patients with knee OA. ${ }^{13-15}$ Here we review the literature that describes thermal neurolysis, and specifically CRFA, in the treatment of knee, hip, and shoulder osteoarthrosis as well as describe the procedural techniques used in our clinic.

\section{Knee Osteoarthritis}

Knee OA accounts for $83 \%$ of all OA cases. ${ }^{16}$ Symptomatic knee OA was estimated to affect $>27$ million U.S. adults. ${ }^{17}$ Risk factors that contribute to knee OA include obesity, diabetes, previous knee trauma (by 3.86 times), old age, and muscle weakness. OA accounts for most knee pain, followed by nondegenerative diseases like posttraumatic, postsurgical, patellofemoral pain syndromes and meniscal, tendon, and ligament injuries. ${ }^{18}$ Of all patients with $\mathrm{OA}, 66 \%$ are obese, and most of the obese patients report that their joint pain prevents them from losing weight. ${ }^{19}$ Common clinical symptoms of knee OA are persistent knee pain, morning stiffness, and reduced function. ${ }^{20,21}$ Patients may have crepitus and painfully limited range of motion. Knee OA pain is typically located anywhere from the distal femur to the proximal tibia. It progressively worsens and is exacerbated with prolonged activity or repetitive bending. ${ }^{22}$

Management for knee OA predominantly addresses pain with the goal to improve function and range of motion. Nonoperative treatment options include activity modification, weight loss, orthotics, medical treatment with nonsteroidal anti-inflammatory drugs (NSAIDs), hyaluronic acid (HA), corticosteroid, and platelet-rich plasma injections. Nonsurgical treatments such as education, exercise, insoles, weight loss, and pain medications were effective in reducing knee pain in knee OA patients at 3 months. ${ }^{23}$ NSAIDs were proven to reduce knee pain by $12 \%$ on the Western Ontario and McMaster Universities Osteoarthritis Index (WOMAC) scale to 6 months for patients who had an 8-year mean duration of OA. ${ }^{24}$ However, many patients use NSAIDs only when needed, rather than continuously, and it is not an ideal sole treatment for knee pain.

Weight loss with unsupervised exercises described in the Look AHEAD study were proven to reduce pain and significantly improve physical function in diabetic overweight and obese adults with knee pain at 12 months. ${ }^{25-27}$ For many patients, though, weight loss is a strenuous process that requires time and resources, to which many patients do not have access. In a prospective study, knee braces significantly improved knee pain at 26 weeks in patients with knee OA $(p<0.05) .{ }^{28}$ However, knee braces can be tedious to apply and maintain. Slightly more invasive procedures for knee $O A$ are corticosteroid injections, which have few systemic adverse events. However, a 2015 Cochrane review concluded that, compared with placebo, clinical benefits of knee corticosteroid injections after 1 week are unclear due to low evidence. $^{29}$

Total knee arthroplasty (TKA) is the most invasive treatment for $\mathrm{OA}$ and one of the most common procedures performed in the United States, averaging 500,000 procedures a year in North America. ${ }^{30}$ Patients who received TKA had significantly greater Knee Injury and OA Outcome Scores (meaning better pain relief) at 12 months, compared with patients who were treated nonsurgically via exercise, insoles, and pain medication. ${ }^{31}$ Skou et al also found a significantly greater number of adverse effects in the TKA group (24 TKA patients versus 6 nonsurgical patients, of 95 total patients) than in the nonsurgical group, with complications such as soft tissue infections, postsurgical stiffness, deep vein thromboses, and chronic pain. ${ }^{31}$ With the rise in obesity rates in the past few decades, knee OA cases will continue to climb, and more patients will need TKAs and pain management. ${ }^{32,33}$

\section{Technique}

The most widespread application of CRFA to the knee targets three genicular nerve branches: the superolateral (SL), superomedial (SM), and inferomedial (IM) genicular nerves (-Fig. 1). Recent evidence suggests that additional ablation of the medial retinacular (MR) branch results in significantly greater improvement in knee $\mathrm{OA}$ pain at 6 months $(p=0.004)$, compared with ablation of only the three genicular nerve branches (-Fig. 1). ${ }^{15,34}$

\section{Diagnostic Nerve Block Procedure}

Approximately 3 to 4 weeks before the CRFA procedure, patients undergo diagnostic genicular nerve blocks under fluoroscopic or, less frequently, ultrasonographic (US) guidance to determine their candidacy for CRFA. Patients lie supine on a fluoroscopic table with the symptomatic knee flexed at 30 degrees. Then, 1 to $2 \mathrm{~mL}$ lidocaine is injected superficially at each of the injection sites, and $25 \mathrm{G}$ or $22 \mathrm{G}$ spinal needles are inserted, targeting the concave transitions of the femoral metadiaphysis and condyles for the SL and SM genicular nerves; the IM genicular nerve is targeted by inserting a spinal needle at the concave transition between the tibial plateau and metadiaphyseal shaft (-Fig. 2). ${ }^{15}$ For patients who undergo the fourth nerve block, the needle is 


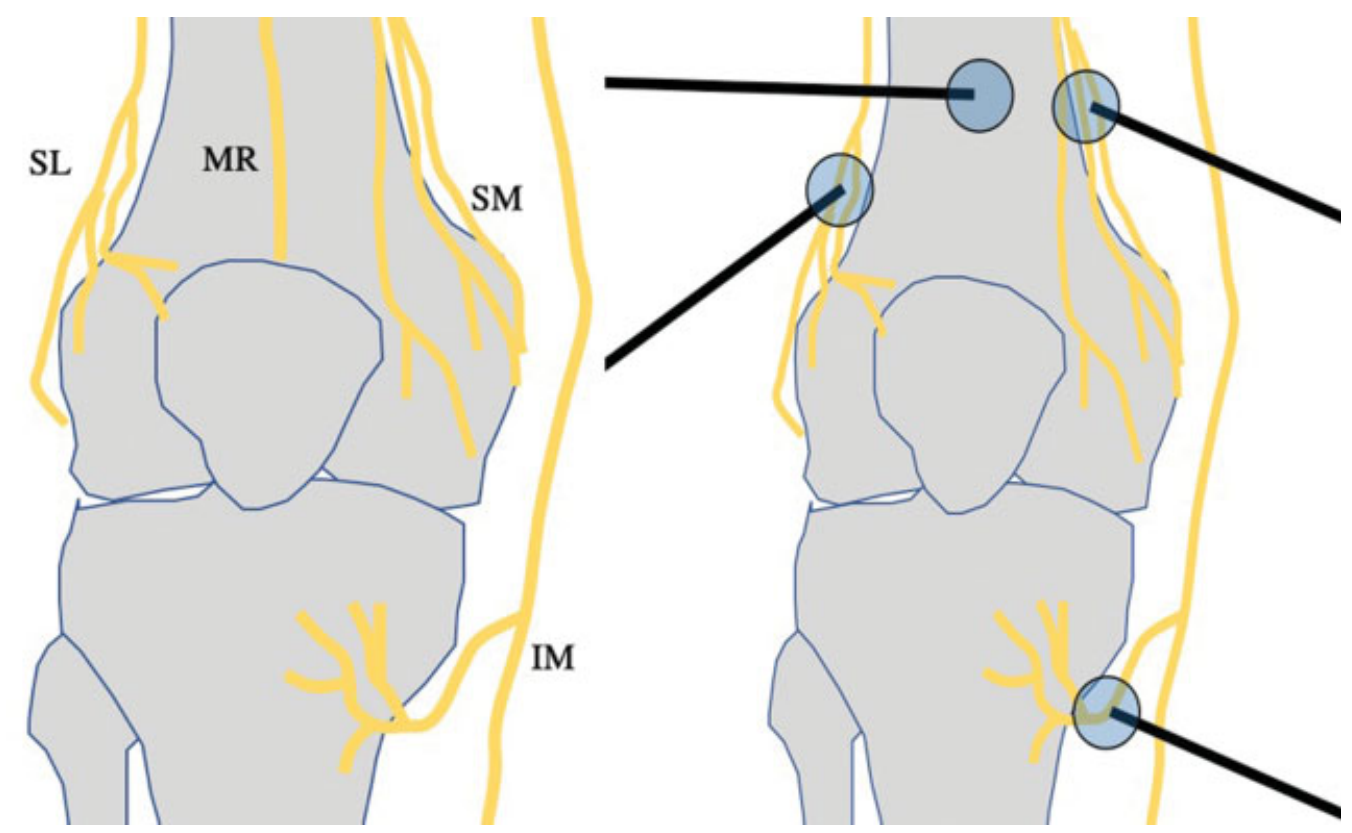

Fig. 1 Schematic illustration showing the respective locations of the genicular nerves around the knee. The three-needle technique uses the introducer needles, targeting the superomedial femoral (SM), superolateral (SL) femoral, and inferomedial (IM) tibial genicular nerves. In the four-needle technique, an additional needle is placed $\sim 3 \mathrm{~cm}$ proximal to the patella to target the medial retinacular nerve (MR).

positioned at the midline anterior distal femoral diaphysis $3 \mathrm{~cm}$ cephalad to the superior patella (-Figure 3). ${ }^{15}$ For each site, $1.0 \mathrm{~mL} 1 \%$ or $2 \%$ lidocaine or $0.25 \%$ bupivacaine is injected. Patients are then assessed after 15 minutes, and if they report at least $50 \%$ of pain reduction during physical examination and ambulation, they are considered candidates for CRFA.

\section{Radiofrequency Ablation Procedure}

Patients arrive for the CRFA procedure 2 to 3 weeks later, and informed consent is obtained. Patients lie supine on the fluoroscopy table, and moderate sedation is achieved with 1 to $2 \mathrm{mg}$ intravenous (IV) midazolam or 25 to $100 \mu \mathrm{g}$ IV fentanyl, as needed. Patients are provided with supplementary oxygen via nasal cannula, and vitals are monitored throughout the procedure. To ensure adequate conduction of the CRFA, a grounding pad is placed ipsilateral to the target joint, avoiding major scar tissue, bony prominences, metal prostheses, electrocardiogram (EKG) electrodes, and areas of possible edema. Then 1 to $2 \mathrm{~mL} 2 \%$ lidocaine is superficially injected at each target site for CRFA. ${ }^{15}$
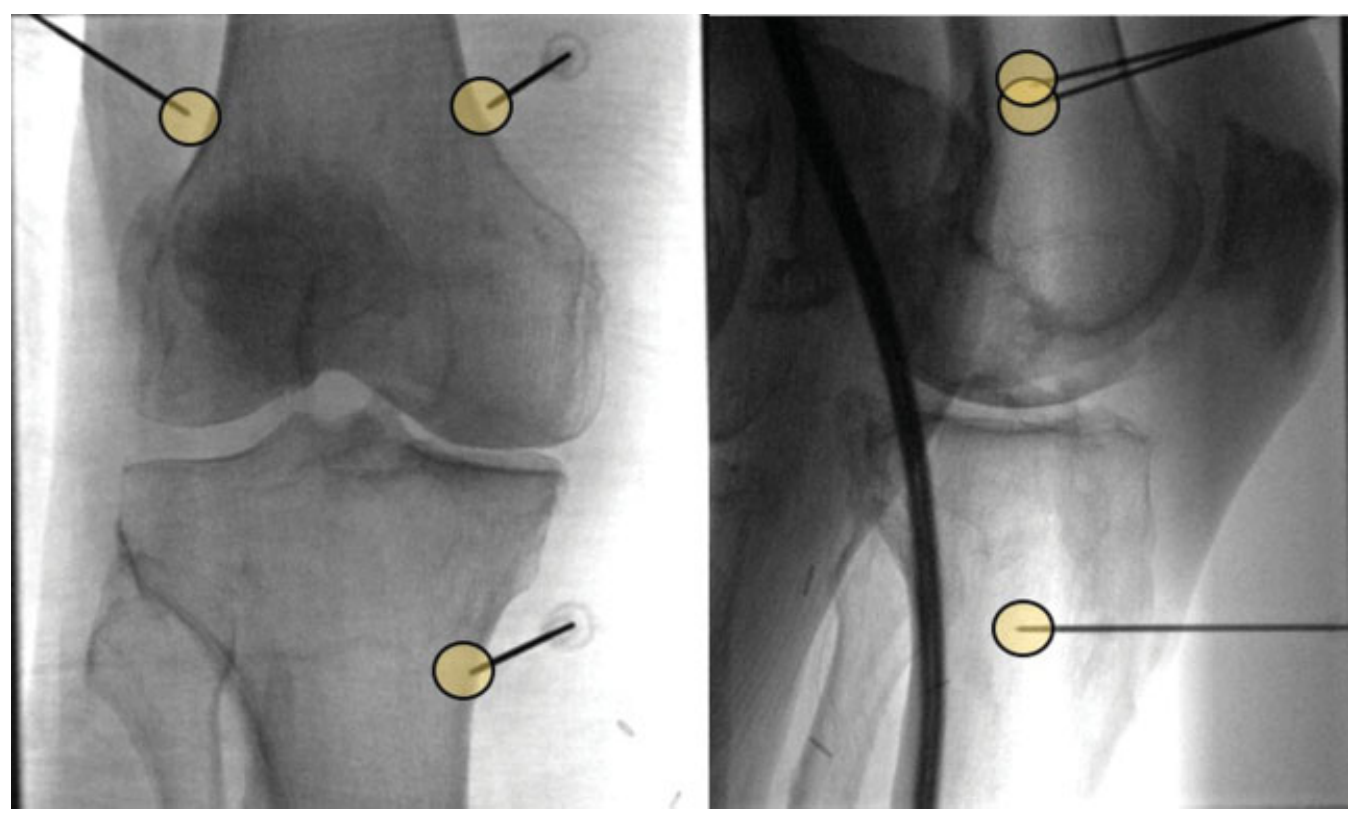

Fig. 2 Three-needle approach targeting the respective genicular nerve locations on the anteroposterior (left) and lateral (right) views. On the lateral view, the guide needles cover at least $50 \%$ of the bone shaft width. 


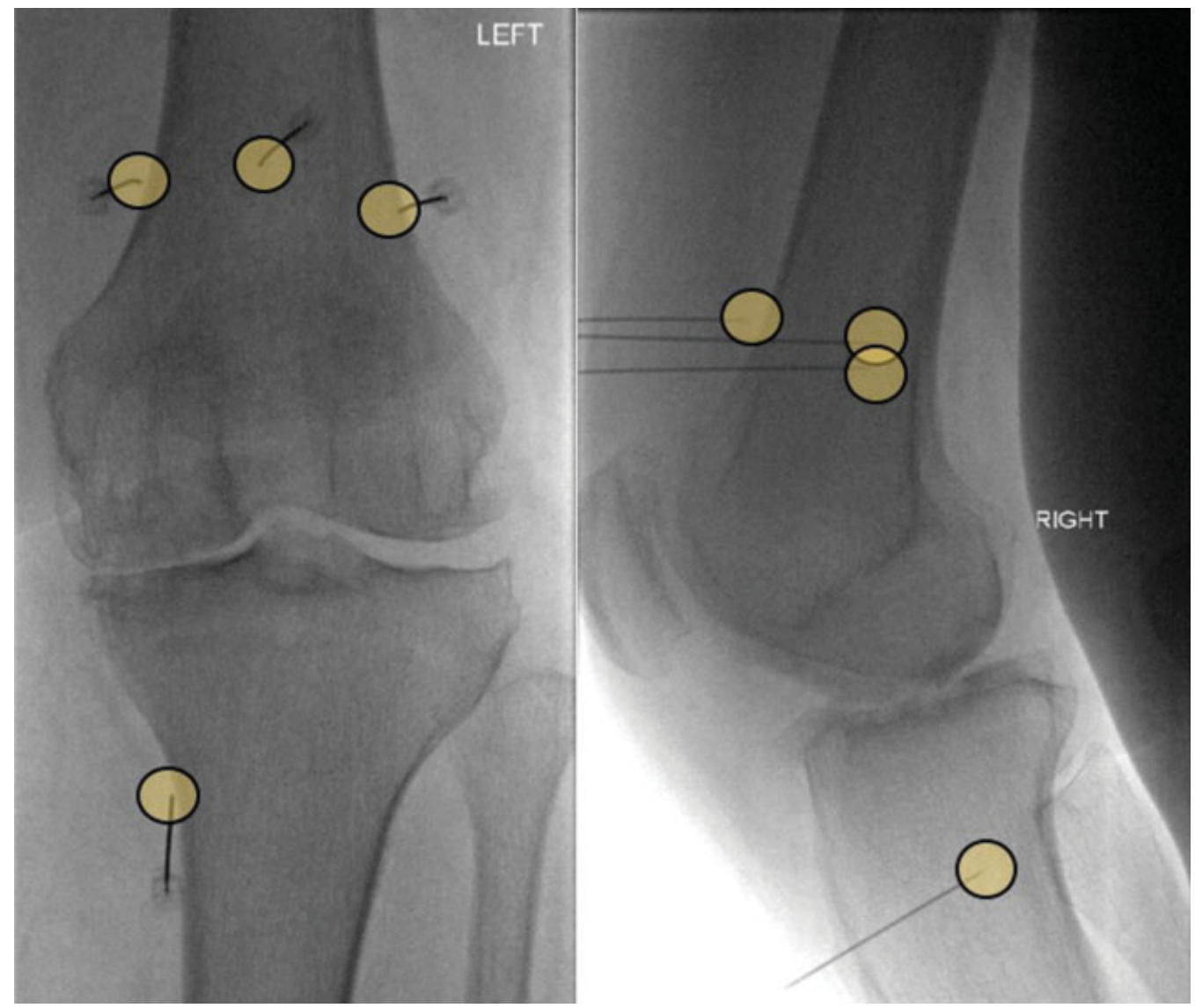

Fig. 3 Four-needle approach targeting the respective genicular nerve locations on the anteroposterior (left) and lateral (right) views.

The sites for CRFA are similar to the sites used for the diagnostic genicular nerve block. The $17 \mathrm{G}$ introducer needles are placed at the SM, SL, MR, and IM nerve locations (-Figs. 2 and 3). An 18G RFA electrode (4mm active tip) is inserted into the introducer needle, and position is confirmed with anteroposterior (AP) and lateral fluoroscopic views. Motor nerve activity is tested and excluded. Local anesthesia with $1 \mathrm{~mL} 2 \%$ lidocaine is injected into the introducer needle. Each nerve is then ablated for $180 \mathrm{sec}-$ onds at $60^{\circ} \mathrm{C}^{15}$

\section{Efficacy of CRFA for Treating Knee OA Pain}

Multiple studies have assessed the efficacy of CRFA in alleviating knee pain secondary to OA. Bellini and Barbieri reported the first nine cases and found significant improvement in perceived pain 1 month after treatment. The baseline visual analog scale (VAS) mean score was $8.0 \pm 1.5$ and decreased to $2 \pm 0.5$ at 1 month posttreatment and to $2.2 \pm 0.2$ at 12 months posttreatment. A noticeable improvement in function was also noted with the WOMAC mean score, which was $88 \pm 1.9$ at baseline, decreased to $20 \pm 2$ at 1 month and 12 months $(20 \pm 1.0) .{ }^{35}$ One of the latest retrospective reviews of 275 geniculate nerve block patients reported a significant decrease of the VAS score from 8.5 to 4.2 after CRFA. In this cohort, $65 \%$ of patients claimed $>50 \%$ pain relief lasting up to 12.5 months, and $77 \%$ of patients had a decrease of $\geq 2$ VAS points 6 months after CRFA. ${ }^{36}$ However, case reports and retrospective studies are subject to selection bias and placebo effects.

CRFA was also compared with corticosteroid injections for the treatment of knee pain in two randomized crossover trials by Davis et al. A total of 151 patients with pain $\geq$ 6 months who did not respond to conservative treatment were enrolled in the studies and randomized to CRFA or intra-articular corticosteroid injection (IACS). ${ }^{37}$ At 6 months, numeric rating scale (NRS) score for CRFA was significantly less than for IACS injections $(2.5 \pm 2.3$ versus $5.9 \pm 2.2$, respectively). The pain reduction $\geq 50 \%$ (from NRS scores) was statistically greater in the CRFA than the IACS group, $74.1 \%$ versus $16.2 \%$, respectively ( $p<0.0001$ ). At 12 months, $65 \%$ of patients who were originally treated with CRFA reported $>50 \%$ pain reduction with a significant 4.2-point drop on NRS (baseline NRS: $7.3 \pm 1.2 ; 12$-month NRS: $3.1 \pm 2.7$ ). CRFA management of knee pain occurs sooner than IACS injections, and its beneficial effects can last up to 12 months. Studying the impact of CRFA is of great interest but has encountered multiple challenges. In the aforementioned study, patients were taking opioids for different reasons (back pain, hip pain, etc.), so it was difficult to assess the duration of opioid use before CRFA treatment, and study cohorts are small. At 18 months, 12 of 25 subjects (48.0\%) continued to report $\geq 50 \%$ pain relief, and 20 patients $(80.0 \%)$ reported improvement from their chronic pain after CRFA. At 24 months, 11 of 18 patients experienced $\geq 50 \%$ pain relief. 
These data imply that the benefits of CRFA may extend up to 24 months in some patients. ${ }^{38}$ No adverse events were reported at 18 and 24 months.

CRFA was also compared with HA injections in a randomized controlled trial. ${ }^{39}$ Chen et al enrolled 177 patients in a randomized crossover study with follow-ups at 1, 3, 6, and 12 months. At 6 months, the CRFA group experienced a significantly greater degree of pain relief compared with HA injections with NRS scores of 2.7 and 5.0, respectively $(p<0.0001)$. From the study's findings at 6 months, CRFA achieved faster pain relief in patients with knee pain compared with HA injections. At 12 months, both treatments demonstrated a significant decrease in NRS score from the 6month mark (CRFA NRS was 2.8 and HA injection NRS was 3.0) $(p<0.0001)$.

These two clinical trials suggest a higher effectiveness of CRFA in treating symptoms related to knee arthritis compared with other conservative measures; however, there is some heterogeneity in the response described for all treatments. Patients are currently deemed candidates for CRFA based on responding successfully to the nerve block with at least $50 \%$ pain decrease used as a discriminator, but there are also nonresponders to CRFA among these patients. ${ }^{15}$ More research is needed to understand the ability of nerve blocks and other patient characteristics to predict response to CRFA, to improve patient pre-selection for this procedure. The pathophysiology of $\mathrm{OA}$ is complex and multifactorial, and it requires a higher order of phenotyping that would significantly reduce trial and error in the clinic and may lead to better nonsurgical outcomes. ${ }^{40}$

\section{Hip Osteoarthritis}

The most common cause of chronic hip pain with advancing age is OA that is also associated with stiffness, restriction of mobility, atrophy of muscles, impaired ambulation, and microinstability. ${ }^{41}$ About 12 to $14.3 \%$ of adults $>60$ years of age have hip pain. ${ }^{42,43}$ Other important causes of chronic pain in the hip include rheumatoid arthritis, acetabular labral tears, osteonecrosis, posttraumatic arthritis, chronic infectious coxarthrosis, avascular necrosis, and persistent postoperative pain following total hip arthroplasty (THA). ${ }^{44}$

Conservative management strategies including education regarding activity and weight loss, physical therapy, assistive devices, and analgesics (acetaminophen, NSAIDs, opioids) are often used to provide pain relief in patients with pain from hip OA, but these interventions confer only temporary benefits and often lack efficacy. ${ }^{45}$ Nonopioid analgesics may have adverse effects, such as gastrointestinal bleeding and renal failure. ${ }^{46}$ Minimally invasive interventional techniques. such as intra-articular injections with corticosteroids, visco-supplementation, and regenerative therapies. often fail to provide long-term pain relief. ${ }^{47,48}$ Patients who do not respond to conservative treatments usually undergo THA, which has a high failure rate (5-15\%), significant cost (average cost of THA and revision hip surgery is $\$ 13,339$ and $\$ 21,224$, respectively), and substantial morbidity including persistent postsurgical pain in 7 to $28 \%$ of patients, and mortality. ${ }^{49-55}$

"Hip pain" is difficult to define topographically. Patients with pain related to pathology in the hip joint present with pain in one or more of these locations: groin, anterolateral thigh, gluteal region, and occasionally referred pain below the knee. Potential pain generators include intra-articular and extra-articular structures, such as ligaments, labrum, cartilage, synovium, bone, bursae, tendons, and nerves. Among the various locations, groin pain is by far the most common, and limitation of internal rotation on physical examination usually suggests intra-articular pathology. ${ }^{56}$

\section{Technique}

The innervation of the hip joint is complex, compartmentalized, and originates from multiple sources. The anteromedial hip is supplied by the articular sensory branches of the obturator nerve, and the anterior hip joint capsule is innervated by the sensory articular branches of the femoral nerve (-Fig. 4). The posterior hip is supplied by the articular sensory branches of the sciatic nerve, and the posteromedial hip joint capsule is innervated by the sensory articular branches of the nerve to the quadratus femoris muscle. Because the anterior capsule has a high concentration of nociceptors and mechanoreceptors, it is the ideal anatomical region for CRFA. ${ }^{57,58}$

\section{Diagnostic Nerve Block Procedure}

Diagnostic nerve blocks are performed to determine candidacy for CRFA. Patients lie supine, and 0.5 to $1.0 \mathrm{~mL}$ lidocaine is injected at two positions: the 12 o'clock position of the acetabular dome to target the femoral sensory nerve branch

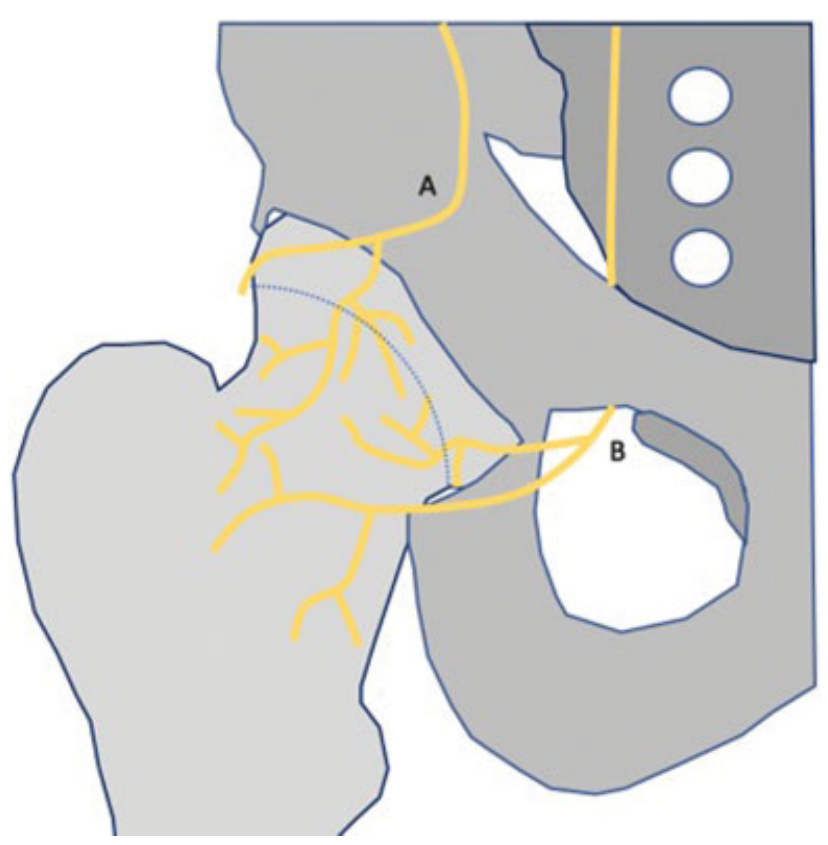

Fig. 4 Schematic illustration of the complex nerve anatomy along the anterior aspect of the hip. The expected anatomical course of the femoral nerve and sensory branches along the superior acetabulum is marked as A. The obturator nerve and its sensory branches is marked as B. 
(es), and the acetabular incisura (junction of the ischium and inferior pubic ramus) to target the obturator branch. If the patient reports at least a $50 \%$ reduction in the subjective Likert scale pain score 15 to 20 minutes after the nerve block procedure, ablation is performed 3 to 4 weeks later. ${ }^{59}$

\section{Radiofrequency Ablation Procedure}

Patients lie supine upon the fluoroscopy table, with the hip, inguinal region, and medial thigh exposed and sterilely prepped. They are moderately sedated with 1 to $2 \mathrm{mg}$ IV midazolam or 25 to $100 \mu \mathrm{g}$ IV fentanyl, as needed, and provided with supplementary oxygen via nasal cannula. To ensure adequate conduction of the CRFA, a grounding pad is placed ipsilateral to the target joint, avoiding major scar tissue, bony prominences, metal prostheses, EKG electrodes, and areas of possible edema. Then 1 to $2 \mathrm{~mL} 1 \%$ lidocaine is injected superficially at each target site for CRFA.

For the obturator nerve, the inguinal ligament serves as the anatomical landmark. First, a detailed evaluation of the femoral neurovascular bundle is performed under US, and the proximal medial thigh skin is marked with a line indicating the location of the major vessels. A scout AP pelvis view is performed to evaluate obturator foramina for symmetry. For maximal ablation, the acetabular incisura is targeted for obturator nerve ablation. The hip, inguinal area, and medial thigh are exposed and sterilely prepared. A 25G needle is inserted posterior to the medial border of the femoral artery and advanced deep to the femoral neurovascular sheath, with great care to avoid the femoral artery, vein, and nerve, until the needle makes bony contact at the acetabular incisura. Local anesthesia is delivered along the tract. The CRFA introducer $(4 \mathrm{~mm}$ active tip) is advanced in a similar fashion and kept in intermittent contact with the anterior ischial surface to avoid the needle traveling superficially. Following aspiration to confirm no blood return, 0.5 to $1 \mathrm{~mL}$ contrast can be injected for correct anatomical confirmation. Motor nerve activity is excluded with testing ( $2 \mathrm{~Hz}$ at $1 \mathrm{~mA}$ ). Once cleared, 0.5 to $1 \mathrm{~mL}$ lidocaine is injected for deep local anesthesia followed by two successive ablations (at the incisura and $3 \mathrm{~mm}$ distal to it) (-Figs. 5 and 6 ).

For the sensory branches of the femoral nerve, the target site for ablation is the 12 o'clock superior acetabular position. The entry site is located $2 \mathrm{~cm}$ lateral to the femoral artery.

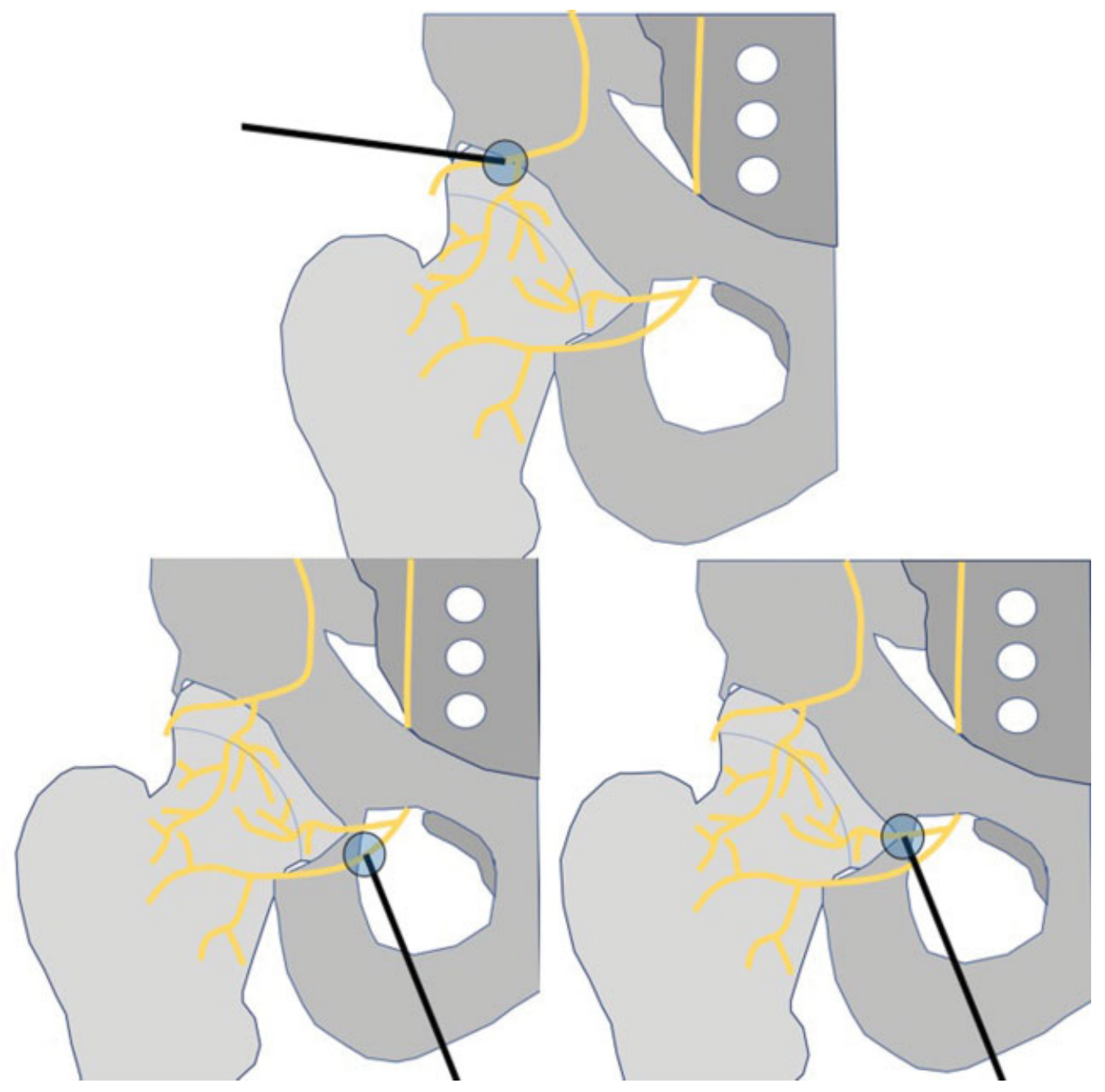

Fig. 5 Top illustration shows proper probe location for the treatment of the femoral nerve branch at $\sim 12$ o'clock in the acetabular dome. Bottom two images show the respective location near the incisura, treating branches of the obturator nerve. 


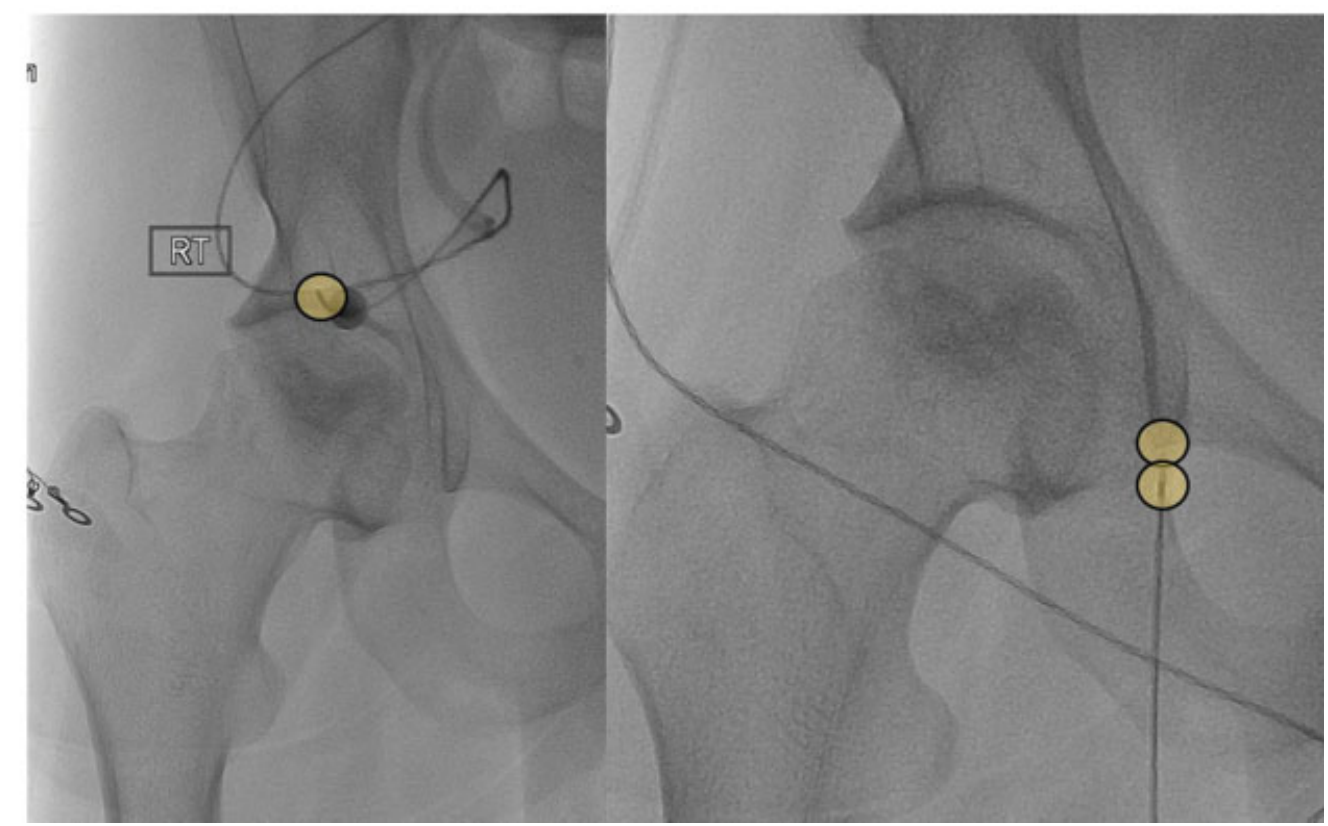

Fig. 6 Hip radiofrequency ablation for the treatment of symptomatic right hip arthritis. Left: Anteroposterior (AP) fluoroscopic image demonstrates placement of a $17 \mathrm{G} 4 \mathrm{~mm}$ active tip probe targeting the location of the femoral nerve articular branch centered at 12 o'clock in the acetabular dome. In this location only one treatment is performed at a set temperature of $60^{\circ} \mathrm{C}$ for 2.5 minutes. Right: AP fluoroscopic image demonstrates placement of a treatment probe targeting the bottom of the incisura in the expected location of the obturator articular branch. Two ablations are performed $3 \mathrm{~mm}$ apart from each other, as shown by the circles.

Superficial local anesthesia is applied to the area, and the needle is inserted until bony contact is made at the osseous acetabulum. The CRFA introducer is inserted along the anesthetized tract, until bony contact is made with the underlying acetabular plate. Motor neuron stimulation is performed and when excluded, 1 to $2 \mathrm{~mL}$ deep local anesthesia is injected. The CRFA probe is placed, and the sensory nerves are ablated (-Figs. $\mathbf{5}$ and $\mathbf{6}$ ).

\section{Efficacy of CRFA for Treating Hip OA Pain}

In a systematic review of 16 publications ( 6 case reports and 7 retrospective and 3 prospective case series) that used TRFA to treat all sources hip pain including OA, TRFA patients had significant reduction in pain at 8 days to 3 years after the procedure; analgesic benefit varied between $30 \%$ and $90 \%$ from baseline scores. ${ }^{60}$ Kapural et al performed 52 fluoroscopic and US-guided CRFA procedures in 23 patients who had hip pain. ${ }^{61}$ They found that significant reduction in hip pain was achieved for up to 320 days after the first ablation and up to 300 days after the second ablation, with VAS score decreasing from $7.61 \pm 1.2$ (baseline) to $2.25 \pm 1.4$ after the second ablation. In a pilot prospective study at our institution (unpublished data), 11 hip pain patients' overall VAS score decreased from $8.3 \pm 1.1$ at baseline to $2.5 \pm 0.5$ at 6 months after CRFA.

Unlike for knee OA, no studies have compared CRFA with other conservative treatments for hip OA. Further research must be performed comparing other conservative modalities with CRFA to further understand this alternative method of treating pain in different clinical settings, such as advanced arthrosis and persistent pain after THA.

\section{Shoulder Osteoarthritis}

The four most common causes of shoulder pain include rotator cuff and glenohumeral disorders, acromioclavicular joint pathology, and referred neck pain. ${ }^{62}$ Shoulder OA is responsible for $4 \%$ of shoulder pain and has severely debilitating symptoms of pain and limited range of motion. ${ }^{63,64}$ As age increases from 40 to 80 years, shoulder OA prevalence increases from $1.8 \%$ to $27.5 \%$, respectively. ${ }^{65}$ Patients who exercise excessively or practice occupations that require over-the-head lifting can also accelerate shoulder OA. Other risk factors include surgeries such as arthroscopic Bankart repairs, fractures, rotator cuff pathology, scapular dysmorphology, inflammatory arthritis, and avascular necrosis of the humeral head. ${ }^{66}$

The goals of conservative treatments are to treat pain and improve range of motion before disuse causes muscle atrophy. Physical therapy is a useful modality that helps patients gain mobility and isometric strength. Medical management with NSAIDs and acetaminophen helps relieve pain and inflammation with relatively great efficacy lasting up to 3 months. ${ }^{67}$ Intra-articular injections for shoulder OA have been studied, but not as extensively as knee and hip OA, and there is no level 1 and 2 evidence. ${ }^{68}$ For instance, Merolla et al compared injections with HA and corticosteroid shots in 84 patients, showing that VAS pain scores significantly decreased from $6.1 \pm 0.9$ to $3.65 \pm 0.9$ at 6 months in the HA group $(p<0.05)$ and a nonsignificant decrease from $6.25 \pm 1.7$ to $5.94 \pm 1.2$ at 6 months in the corticosteroid group $(p=0.069) .{ }^{69}$ A more recent prospective study demonstrated a significant decrease in shoulder pain at 6 months 


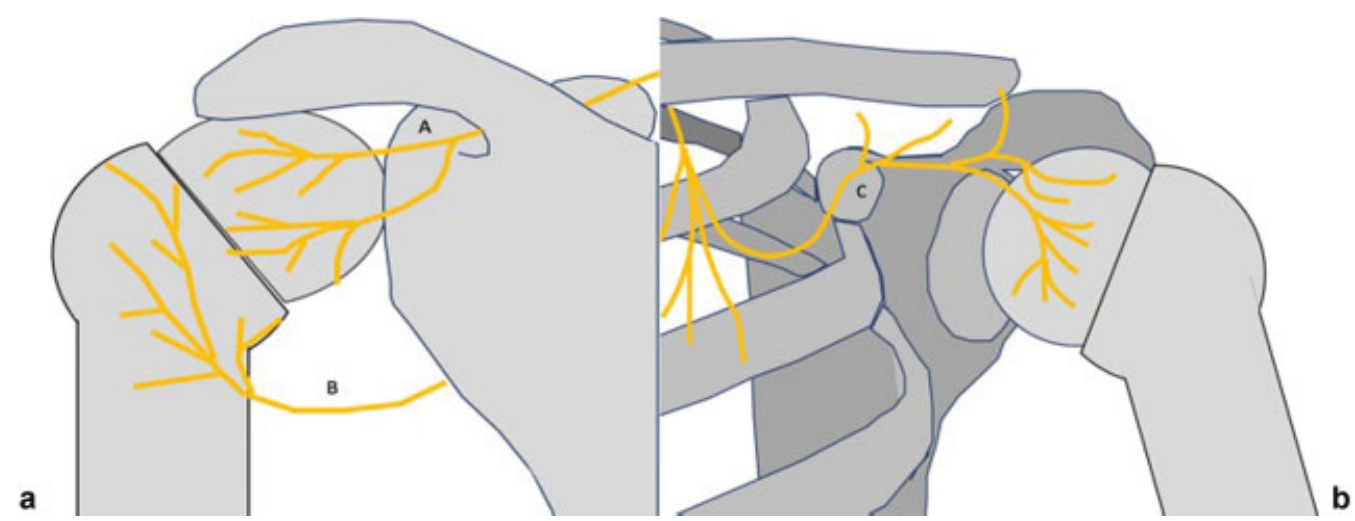

Fig. 7 Schematics demonstrate the respective (a) posterior and (b) anterior nerves about the shoulder joint. The anatomical course of the suprascapular nerve and branches along the superior aspect of the glenoid rim is marked as A. B depicts the course of the axillary nerve branches along the greater tuberosity of the humerus. The course of the lateral pectoral major branch in the region of the coracoid process along the anterior shoulder is marked as $C$.

after HA injection with physical therapy, compared with patients treated with physical therapy only. ${ }^{70}$

\section{Technique}

The innervation of the shoulder joint is intricate. The suprascapular nerve innervates most of the posterior shoulder capsule, subacromial bursa, and coracoacromial and acromioclavicular ligaments ( - Fig. 7 ). The axillary nerve innervates the posterior inferior and lateral aspects of the glenohumeral capsule. The lateral pectoral nerve innervates the anterosuperior region of the shoulder (anterior border of the subacromial bursa, the coracoacromial ligaments, and the anterior joint capsule). The highest density of nociceptors and mechanoreceptors was reported in the subacromial bursa and anterior glenohumeral capsule/ligaments. ${ }^{71}$

\section{Diagnostic Nerve Block Procedure}

Patients undergo diagnostic nerve injection at three target sites to determine candidacy for CRFA (-Fig. 7). The suprascapular nerve is targeted along the posterior superior third of the glenoid rim, $\sim 2$ to $3 \mathrm{~mm}$ away from the articular surface ( $\mathbf{F i g}$. 7a, A), the axillary nerve is targeted at the inferolateral border of the greater tuberosity

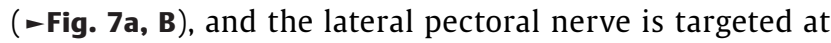
the midpoint of the coracoid process ( $\mathbf{- F i g .} \mathbf{7 b}, \mathbf{C}$ ). If the patient reports at least $50 \%$ reduction in the subjective Likert scale pain score 15 to 20 minutes after the nerve block procedure, ablation is performed 3 to 4 weeks later. $^{59}$

\section{Radiofrequency Ablation Procedure}

To target the posterior shoulder, the patient is positioned in the prone position. The hand ipsilateral to the affected shoulder is internally rotated (pronated forearm). The grounding pad is applied to the patient's ipsilateral flank. The patient's posterior shoulder is sterilely prepped for the procedure.

The suprascapular nerve is targeted at two different sites. To target the first suprascapular nerve site, the $\mathrm{C}$-arm is then rotated 15 to 45 degrees from the AP position toward the affected shoulder to find the posterior border of the glenoid process to profile the glenohumeral joint. A 15-degree caudal tilt is added to visualize the glenoid in full view ( - Figs. 8a and 9a). Local anesthesia is then administered in the expected location of the suprascapular nerve, at the junction of the upper and middle thirds of the glenoid rim, near the articular surface, lateral and superior to the spinoglenoid notch. 17G introducer is inserted until it contacts the superior glenoid rim 2 to $3 \mathrm{~mm}$ medial to the articular surface to avoid inserting the needle into the joint space. The introducer stylet is removed, the CRFA probe (2mm active tip) is placed, and motor neurons are excluded via nerve stimulation. The rotator cuff muscles should also be observed for twitching or movement. If there are any positive responses to motor nerve stimulation, the introducer should be moved laterally, increasing the distance from the suprascapular motor trunk. After motor nerve exclusion, deep local anesthesia is injected, and the sensory nerve is ablated for 150 seconds. The second suprascapular nerve site is 3 to $4 \mathrm{~mm}$ inferior to the first position (posterior osseous rim of
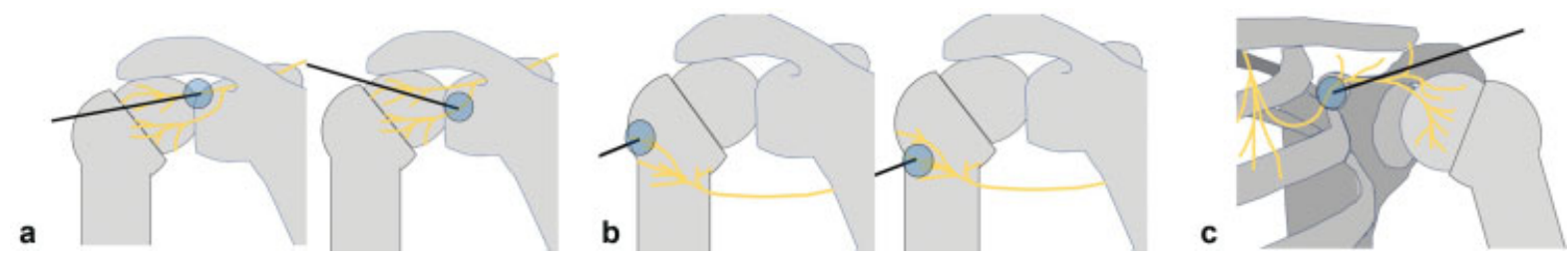

Fig. 8 (a) Radiofrequency ablation probe placements for the treatment of the suprascapular nerve branches. (b) Needle placements at the branches of the axillary nerve. (c) Treatment of the lateral pectoral nerve. 

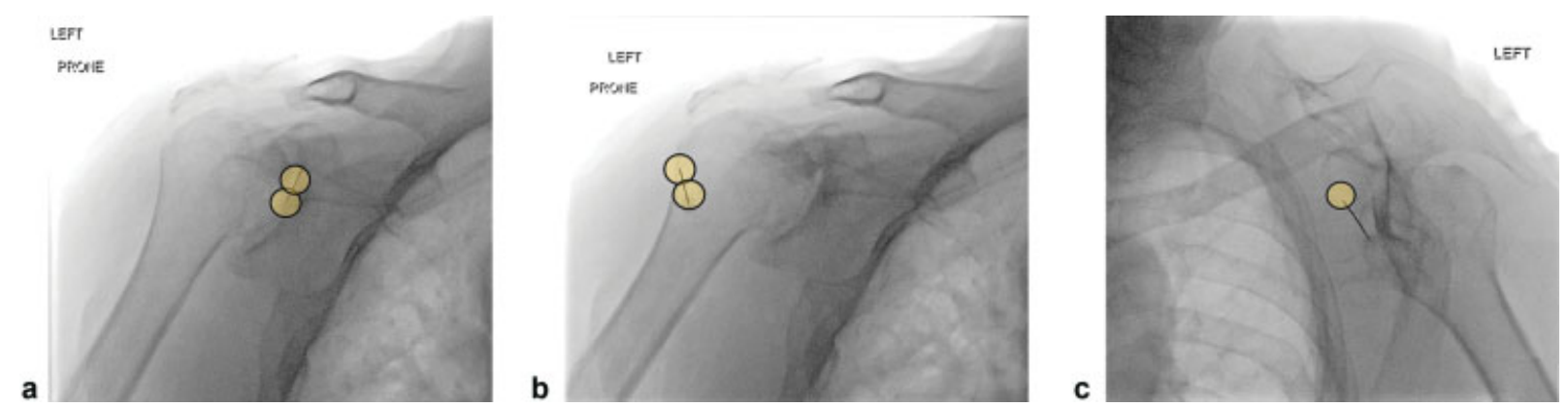

Fig. 9 Shoulder radiofrequency ablation. (a) Location of the respective 22G spinal needles targeting the suprascapular nerve articular branch at the upper third aspect of the glenoid 1 to $2 \mathrm{~mm}$ away from the articular surface. (b) Axillary branch at the inferior aspect of the humeral head greater tuberosity while the patient lies prone along the posterior aspect of the shoulder shown by the circles. (c) Then the patient is turned around and while lying on the supine position, the lateral pectoral nerve block is targeted at the center-inferior aspect of the coracoid process.

the glenoid fossa, lateral to the spinoglenoid notch) (-Figs. $\mathbf{8 a}$ and $\mathbf{9 a}$ ). The same skin entry site should be used, repositioning the introducer, and the same steps are taken to ablate the nerve for 150 seconds.

There are two sites for the axillary nerve. The C-arm angles are the same, but the image is centered over the humeral head. For the first axillary nerve site, local anesthesia is injected superficially. The introducer is inserted at the outermost aspect of the greater tuberosity above the level of the surgical neck (-Figs. $8 \mathbf{b}$ and $\mathbf{9 b}$ ). The introducer is advanced until it contacts the cortex. Motor nerve stimulation is initiated while also observing for movement in the deltoid and teres minor muscles. If detected, the introducer must be repositioned cranially and laterally to distance it from the axillary motor nerve trunk. Once excluded, the sensory nerve can be ablated. For the second axillary nerve site, the probe is removed and the stylet replaced. Using the same skin entry site, the introducer is positioned 3 to $4 \mathrm{~mm}$ inferior to the greater tubercle, where it tapers into the humeral diaphysis while remaining on the most lateral humeral border. The same steps are taken to ablate this second axillary nerve ( - Figs. $\mathbf{8 b}$ and $\mathbf{9 b}$ ).

There is only one ablation site for the lateral pectoral nerve ( - Figs. $\mathbf{8 c}$ and $\mathbf{9 c}$ ). The patient is turned from prone to supine position on the table. The patient's anterior shoulder is sterilely prepped. The grounding pad's adherence should be confirmed again on the patient's ipsilateral flank, in case it was moved or dislodged while the patient was being repositioned. To visualize the shoulder girdle, the procedure begins with the C-arm rotated 15 degrees toward the affected shoulder from the posteroanterior view, and then rotated medially until the coracoid process superimposes medially upon the glenohumeral joint. Then a 15-degree cephalic tilt is applied to give the coracoid process a "thumbs-down" appearance. The introducer is inserted at the midpoint of the coracoid process and advanced until it contacts the cortex. Because the sensory branches of the lateral pectoral nerve are superficial, the introducer must be at least $2 \mathrm{~cm}$ deep to avoid skin burns during ablation. Motor neuron stimulation is performed while observing the pectoralis major muscle. If there is a positive response, the introducer should be moved laterally to avoid the lateral pectoral motor trunks. Once motor nerves are excluded, deep local anesthesia is injected, and ablation is performed.

\section{Efficacy of CRFA for Treating Shoulder OA Pain}

Little evidence supports CRFA for shoulder pain from OA. Eckmann et al performed a retrospective study that investigated CRFA that targeted the suprascapular, axillary, and lateral pectoral nerve in patients with chronic intractable shoulder pain. ${ }^{72}$ At the first follow-up visit of 3 months, all 10 patients reported a decrease in mean NRS pain score from 6.0 to $1.1(p=0.001)$. Six of 10 patients who were followed until 6 months reported $>50 \%$ pain relief at the second time point. Four of these patients had shoulder $\mathrm{OA}$, and the remaining two patients had rotator cuff tendinopathy.

Other studies have looked at TRFA for shoulder pain, but not shoulder pain from OA specifically. Gabrhelik et al retrospectively studied 28 patients who received pulsed TRFA to the suprascapular nerve and compared local anesthetic and pulsed TRFA with and without corticosteroid injection. ${ }^{73}$ They found that both groups had significant reduction in VAS scores at 6 months (corticosteroid group: 4.6; 95\% confidence interval [CI], 3.7-5.6 versus 2.6 ; $95 \% \mathrm{CI}, 1.5-$ 3.6; non-corticosteroid group: 5.1 ; $95 \% \mathrm{CI}, 4.5-6.7$ versus 1.6 ; $95 \% \mathrm{CI}, 0.5-2.6$ ) with no statistically significant difference between the corticosteroid and non-corticosteroid group ( $p>0.05)$. From the current literature, little evidence supports CRFA treatment for shoulder pain from OA. More studies are needed to evaluate its efficacy for shoulder $\mathrm{OA}$ and other etiologies of shoulder pain, and also to compare its efficacy with other treatment modalities including HA and corticosteroid injections.

\section{Conclusion}

Thermal ablation techniques such as CRFA have evolved as alternative treatment methodology for moderate to severe forms of symptomatic knee OA among patients who have failed conservative treatments. CRFA treatment of knee pain is supported by prospective clinical trials that report sustained efficacy for up to 2 years, which is longer than conventional treatments such as corticosteroid and HA injections. The limited number of studies evaluating CRFA for the treatment of symptomatic advanced hip OA suggests 
benefit for reducing pain. However, no study has directly compared CRFA with other conservative modalities in this setting. The limited experience of CRFA applied to the treatment of general shoulder pain suggests potential utility in applications related to shoulder OA pain, although prospective clinical trials in patients with shoulder $O A$ are needed to understand the extent and duration of pain relief. OA remains a growing problem that will affect many adults, and CRFA is a promising tool that will likely be used with other treatment options in the management of symptomatic OA.

\section{Conflict of Interest}

None declared.

\section{References}

1 Cui A, Li H, Wang D, Zhong J, Chen Y, Lu H. Global, regional prevalence, incidence and risk factors of knee osteoarthritis in population-based studies. EClinicalMedicine 2020; Available at: https://www.thelancet.com/journals/eclinm/article/PIIS25895370(20)30331-X/fulltext

2 Murphy LB, Cisternas MG, Pasta DJ, Helmick CG, Yelin EH. Medical expenditures and earnings losses among US adults with arthritis in 2013. Arthritis Care Res (Hoboken) 2018;70(06):869-876

3 McGrory BJ, Weber KL, Jevsevar DS, Sevarino K. Surgical management of osteoarthritis of the knee: evidence-based guideline. J Am Acad Orthop Surg 2016;24(08):e87-e93

4 Ward ZJ, Bleich SN, Cradock AL, et al. Projected U.S. state-level prevalence of adult obesity and severe obesity. N Engl J Med 2019; 381(25):2440-2450

5 Chillemi C, Franceschini V. Shoulder osteoarthritis. Arthritis (Egypt) 2013;2013:370231

6 Ayhan E, Kesmezacar H, Akgun I. Intraarticular injections (corticosteroid, hyaluronic acid, platelet rich plasma) for the knee osteoarthritis. World J Orthop 2014;5(03):351-361

7 Kompel AJ, Roemer FW, Murakami AM, Diaz LE, Crema MD, Guermazi A. Intra-articular corticosteroid injections in the hip and knee: perhaps not as safe as we thought? Radiology 2019;293(03):656-663

8 Wernecke C, Braun HJ, Dragoo JL. The effect of intra-articular corticosteroids on articular cartilage: a systematic review. Orthop J Sports Med 2015;3(05):2325967115581163

9 Kloppenburg M, Berenbaum F. Osteoarthritis year in review 2019: epidemiology and therapy. Osteoarthritis Cartilage 2020;28(03): 242-248

10 Neogi T. The epidemiology and impact of pain in osteoarthritis. Osteoarthritis Cartilage 2013;21(09):1145-1153

11 Wylde V, Sayers A, Lenguerrand E, et al. Preoperative widespread pain sensitization and chronic pain after hip and knee replacement: a cohort analysis. Pain 2015;156(01):47-54

12 Hong K, Georgiades C. Radiofrequency ablation: mechanism of action and devices. J Vasc Interv Radiol 2010;21(8, Suppl): S179-S186

13 Gonzalez FM. Cooled radiofrequency genicular neurotomy. Tech Vasc Interv Radiol 2020;23(04):100706

14 Rojhani S, Qureshi Z, Chhatre A. Water-cooled radiofrequency provides pain relief, decreases disability, and improves quality of life in chronic knee osteoarthritis. Am J Phys Med Rehabil 2017;96(01):e5-e8

15 Wong PK, Kokabi N, Guo Y, et al. Safety and efficacy comparison of three- vs four-needle technique in the management of moderate to severe osteoarthritis of the knee using cooled radiofrequency ablation. Skeletal Radiol 2021;50(04):739-750

16 Vos T, Flaxman AD, Naghavi M, et al. Years lived with disability (YLDs) for 1160 sequelae of 289 diseases and injuries 1990-2010: a systematic analysis for the Global Burden of Disease Study 2010. Lancet 2012;380(9859):2163-2196
17 Lawrence RC, Felson DT, Helmick CG, et al; National Arthritis Data Workgroup. Estimates of the prevalence of arthritis and other rheumatic conditions in the United States. Part II. Arthritis Rheum 2008;58(01):26-35

18 Jones BQ Covey CJ, Sineath MH Jr. Nonsurgical management of knee pain in adults. Am Fam Physician 2015;92(10):875-883

19 Shih M, Hootman JM, Kruger J, Helmick CG. Physical activity in men and women with arthritis National Health Interview Survey, 2002. Am J Prev Med 2006;30(05):385-393

20 Heidari B. Knee osteoarthritis prevalence, risk factors, pathogenesis and features: Part I. Caspian J Intern Med 2011;2(02): 205-212

21 Zhang W, Doherty M, Peat G, et al. EULAR evidence-based recommendations for the diagnosis of knee osteoarthritis. Ann Rheum Dis 2010;69(03):483-489

22 Hsu H, Siwiec RM. Knee osteoarthritis. Treasure Island, FL: StatPearls Publishing; 2021. Available at: www.ncbi.nlm.nih.gov/books/NBK507884/

23 Skou ST, Roos EM, Simonsen O, et al. The efficacy of non-surgical treatment on pain and sensitization in patients with knee osteoarthritis: a pre-defined ancillary analysis from a randomized controlled trial. Osteoarthritis Cartilage 2016;24(01):108-116

24 Puljak L, Marin A, Vrdoljak D, Markotic F, Utrobicic A, Tugwell P. Celecoxib for osteoarthritis. Cochrane Database Syst Rev 2017;5 (05):CD009865

25 Foy CG, Lewis CE, Hairston KG, et al; Look AHEAD Research Group. Intensive lifestyle intervention improves physical function among obese adults with knee pain: findings from the Look AHEAD trial. Obesity (Silver Spring) 2011;19(01):83-93

26 Wadden TA, West DS, Delahanty L, et al; Look AHEAD Research Group. The Look AHEAD study: a description of the lifestyle intervention and the evidence supporting it. Obesity (Silver Spring) 2006;14(05):737-752

27 DeRogatis M, Anis HK, Sodhi N, et al. Non-operative treatment options for knee osteoarthritis. Ann Transl Med 2019;7(Suppl 7):S245

28 Yu SP, Williams M, Eyles JP, Chen JS, Makovey J, Hunter DJ. Effectiveness of knee bracing in osteoarthritis: pragmatic trial in a multidisciplinary clinic. Int J Rheum Dis 2016;19(03): 279-286

29 Jüni P, Hari R, Rutjes AW, et al. Intra-articular corticosteroid for knee osteoarthritis. Cochrane Database Syst Rev 2015;(10): CD005328

30 Cram P, Lu X, Kates SL, Singh JA, Li Y, Wolf BR. Total knee arthroplasty volume, utilization, and outcomes among Medicare beneficiaries, 1991-2010. JAMA 2012;308(12):1227-1236

31 Skou ST, Roos EM, Laursen MB, et al. A randomized, controlled trial of total knee replacement. N Engl J Med 2015;373(17): 1597-1606

32 Agha M, Agha R. The rising prevalence of obesity: part A: impact on public health. Int J Surg Oncol (N Y) 2017;2(07):e17

33 Kurtz S, Ong K, Lau E, Mowat F, Halpern M. Projections of primary and revision hip and knee arthroplasty in the United States from 2005 to 2030. J Bone Joint Surg Am 2007;89(04):780-785

34 Franco CD, Buvanendran A, Petersohn JD, Menzies RD, Menzies LP. Innervation of the anterior capsule of the human knee: implications for radiofrequency ablation. Reg Anesth Pain Med 2015;40 (04):363-368

35 Bellini M, Barbieri M. Cooled radiofrequency system relieves chronic knee osteoarthritis pain: the first case-series. Anaesthesiol Intensive Ther 2015;47(01):30-33

36 Kapural L, Lee N, Neal K, Burchell M. Long-term retrospective assessment of clinical efficacy of radiofrequency ablation of the knee using a cooled radiofrequency system. Pain Physician 2019; 22(05):489-494

37 Davis T, Loudermilk E, DePalma M, et al. Prospective, multicenter randomized, crossover clinical trial comparing the safety and effectiveness of cooled radiofrequency ablation with 
corticosteroid injection in the management of knee pain from osteoarthritis. Reg Anesth Pain Med 2018;43(01):84-91

38 Hunter C, Davis T, Loudermilk E, Kapural L, DePalma M. Cooled radiofrequency ablation treatment of the genicular nerves in the treatment of osteoarthritic knee pain: 18- and 24-month results. Pain Pract 2020;20(03):238-246

39 Chen AF, Khalouf F, Zora K, et al. Cooled radiofrequency ablation provides extended clinical utility in the management of knee osteoarthritis: 12-month results from a prospective, multi-center, randomized, cross-over trial comparing cooled radiofrequency ablation to a single hyaluronic acid injection. BMC Musculoskelet Disord 2020;21(01):363

40 Deveza LA, Nelson AE, Loeser RF. Phenotypes of osteoarthritis: current state and future implications. Clin Exp Rheumatol 2019; 37(5, Suppl 120):64-72

41 Zacher J, Gursche A. 'Hip' pain. Best Pract Res Clin Rheumatol 2003;17(01):71-85

42 Cecchi F, Mannoni A, Molino-Lova R, et al. Epidemiology of hip and knee pain in a community based sample of Italian persons aged 65 and older. Osteoarthritis Cartilage 2008;16(09):1039-1046

43 Lespasio MJ, Sultan AA, Piuzzi NS, et al. Hip osteoarthritis: a primer. Perm J 2018;22:17-084

44 Battaglia PJ, D’Angelo K, Kettner NW. Posterior, lateral, and anterior hip pain due to musculoskeletal origin: a narrative literature review of history, physical examination, and diagnostic imaging. J Chiropr Med 2016;15(04):281-293

45 Zhang W, Doherty M, Arden N, et al; EULAR Standing Committee for International Clinical Studies Including Therapeutics (ESCISIT) EULAR evidence based recommendations for the management of hip osteoarthritis: report of a task force of the EULAR Standing Committee for International Clinical Studies Including Therapeutics (ESCISIT). Ann Rheum Dis 2005;64(05):669-681

46 Crawford DC, Miller LE, Block JE. Conservative management of symptomatic knee osteoarthritis: a flawed strategy? Orthop Rev (Pavia) 2013;5(01):e2

47 Flanagan J, Casale FF, Thomas TL, Desai KB. Intra-articular injection for pain relief in patients awaiting hip replacement. Ann $\mathrm{R}$ Coll Surg Engl 1988;70(03):156-157

48 Lai WC, Arshi A, Wang D, et al. Efficacy of intraarticular corticosteroid hip injections for osteoarthritis and subsequent surgery. Skeletal Radiol 2018;47(12):1635-1640

49 Hip and Knee Replacements: Canadian Joint Replacement Registry 2013. May 6,. 2017; Available at: https://secure.cihi.ca/estore/ productFamily.htm?pf $=$ PFC2209\&lang $=$ en\&media $=0$

50 Belmont PJ Jr, Powers CC, Beykirch SE, Hopper RH Jr, Engh CA Jr, Engh CA. Results of the anatomic medullary locking total hip arthroplasty at a minimum of twenty years. A concise follow-up of previous reports. J Bone Joint Surg Am 2008;90(07):1524-1530

51 Beswick AD, Wylde V, Gooberman-Hill R, Blom A, Dieppe P. What proportion of patients report long-term pain after total hip or knee replacement for osteoarthritis? A systematic review of prospective studies in unselected patients. BMJ Open 2012;2 (01):e000435

52 Grayson CW, Decker RC. Total joint arthroplasty for persons with osteoarthritis. PM R 2012;4(5, Suppl):S97-S103

53 Nikolajsen L, Brandsborg B, Lucht U, Jensen TS, Kehlet H. Chronic pain following total hip arthroplasty: a nationwide questionnaire study. Acta Anaesthesiol Scand 2006;50(04):495-500

54 Antoniou J, Martineau PA, Filion KB, et al. In-hospital cost of total hip arthroplasty in Canada and the United States.J Bone Joint Surg Am 2004;86(11):2435-2439
55 Crowe JF, Sculco TP, Kahn B. Revision total hip arthroplasty: hospital cost and reimbursement analysis. Clin Orthop Relat Res 2003;(413):175-182

56 Poultsides LA, Bedi A, Kelly BT. An algorithmic approach to mechanical hip pain. HSS J 2012;8(03):213-224

57 National Center for Health Statistics. Drug overdose deaths in the United States, 1999-2018 [Data Brief no. 356]. Hyattsville, MD: NCHS; 2020

58 Laumonerie P, Dalmas Y, Tibbo ME, et al. Sensory innervation of the hip joint and referred pain: a systematic review of the literature. Pain Med 2021;22(05):1149-1157

59 Cepeda MS, Africano JM, Polo R, Alcala R, Carr DB. What decline in pain intensity is meaningful to patients with acute pain? Pain 2003;105(1-2):151-157

60 Kumar P, Hoydonckx Y, Bhatia A. A review of current denervation techniques for chronic hip pain: anatomical and technical considerations. Curr Pain Headache Rep 2019;23(06):38

61 Kapural L, Jolly S, Mantoan J, Badhey H, Ptacek T. Cooled radiofrequency neurotomy of the articular sensory branches of the obturator and femoral nerves-combined approach using fluoroscopy and ultrasound guidance: technical report, and observational study on safety and efficacy. Pain Physician 2018;21(03):279-284

62 Mitchell C, Adebajo A, Hay E, Carr A. Shoulder pain: diagnosis and management in primary care. BMJ 2005;331(7525):1124-1128

63 Cadogan A, Laslett M, Hing WA, McNair PJ, Coates MH. A prospective study of shoulder pain in primary care: prevalence of imaged pathology and response to guided diagnostic blocks. BMC Musculoskelet Disord 2011;12:119

64 Millett PJ, Gobezie R, Boykin RE. Shoulder osteoarthritis: diagnosis and management. Am Fam Physician 2008;78(05):605-611

65 Ibounig T, Simons T, Launonen A, Paavola M. Glenohumeral osteoarthritis: an overview of etiology and diagnostics. Scand J Surg 2020; July 14 (Epub ahead of print)

66 Kavaja L, Pajarinen J, Sinisaari I, et al. Arthrosis of glenohumeral joint after arthroscopic Bankart repair: a long-term follow-up of 13 years. J Shoulder Elbow Surg 2012;21(03):350-355

67 Guyot P, Pandhi S, Nixon RM, Iqbal A, Chaves RL, Andrew Moore R. Efficacy and safety of diclofenac in osteoarthritis: results of a network meta-analysis of unpublished legacy studies. Scand J Pain 2017; 16:74-88

68 Gross C, Dhawan A, Harwood D, Gochanour E, Romeo A. Glenohumeral joint injections: a review. Sports Health 2013;5(02): 153-159

69 Merolla G, Sperling JW, Paladini P, Porcellini G. Efficacy of Hylan G-F 20 versus 6-methylprednisolone acetate in painful shoulder osteoarthritis: a retrospective controlled trial. Musculoskelet Surg 2011;95(03):215-224

70 Di Giacomo G, de Gasperis N. Hyaluronic acid intra-articular injections in patients affected by moderate to severe glenohumeral osteoarthritis: a prospective randomized study. Joints 2017;5 (03):138-142

71 Eckmann MS, Bickelhaupt B, Fehl J, et al. Cadaveric study of the articular branches of the shoulder joint. Reg Anesth Pain Med 2017;42(05):564-570

72 Eckmann MS, Johal J, Bickelhaupt B, et al. Terminal sensory articular nerve radiofrequency ablation for the treatment of chronic intractable shoulder pain: a novel technique and case series. Pain Med 2020;21(04):868-871

73 Gabrhelik T, Michalek P, Adamus M, Mikova M, Dolecek L. Effect of pulsed radiofrequency therapy on the suprascapular nerve in shoulder pain of various aetiology. Ir J Med Sci 2010;179(03):369-373 